\title{
A novel data fusion method for infrared and visible images based on NSCT and Gaussian statistical estimation
}

\author{
by Q. Zhang* and X. Maldague* \\ * Univ. Laval, Local 1300, 1065 Avenue de la Médecine, G1V 0A6, Quebec, QC, Canada, \\ qiong.zhang.1@ulaval.ca
}

\begin{abstract}
A novel fusion algorithm for infrared and visible images based on non-subsampled contourlet transform (NSCT) and generalized Gaussian probability density function (GG-PDF) estimation is proposed. First, the source infrared and visible images are decomposed by NSCT to obtain the low and high frequency subband coefficients, respectively. Afterwards, an improved average gradient based fusion rule is applied to fuse the low-frequency coefficients, whereas, the GG-PDF estimation model is introduced to estimate the salience of the high-frequency coefficients and then make a decision of selection of weight factors. Then, the final fusion image is obtained by the inverse NSCT. Experiments prove the advantages of the proposed fusion approach.
\end{abstract}

\section{Introduction}

In the past decades, numerous imaging sensors have been applied to obtain the target or its complementary information. Infrared (IR) images have fine radiometric resolution and can provide alternative characteristics different from visible images, but the IR image sensor is sensitive and dependent on thermal differences in the surrounding environment. The visible image can provide more detailed information of the scene, but cannot reflect inner mechanisms of the target or background. To achieve a comprehensive description considering the optimum visual and characteristic effect, image fusion technique is implemented to merge the IR and visible images.

There have been varieties of different transform-based IR-visible image fusion approaches, e. g. wavelet-based, Curvelet-based, Contourlet-based, etc. fusion methods. However, they cannot solve the problem of information loss, such as edges and curves, which contain a lot of detail information. On the basis of Contourlet transform, the nonsubsampled contourlet transform (NSCT) was proposed by Arthur L. Cunba [1]. It can deal with the fusion processing evidently better, because it captures edges and textures of the image, fits with rich detail and multi-sensor image orientation information, and it is translation-invariant. Thus, the image quality can be kept very well to have eliminated the ringing effect and shake phenomenon.

In this paper, we present a novel image fusion approach employing NSCT and generalized Gaussian probability density function (GG-PDF) [2] to obtain better fusion results. The NSCT decomposes images into low and high frequency subbands for the IR and visible images, respectively. The low-frequency coefficients are fused by an improved regional average gradient fusion rule, whereas, for the high-frequency coefficients, the GG-PDF method is used to estimate the weights of salience from the coefficients of IR and visible images as a first step, then a selection fusion rule is utilized to fuse the corresponding coefficients. Finally, the fused low and high coefficients are reconstructed by inverse NSCT method to obtain the final fusion image.

\section{Image Fusion Approach}

\subsection{Low-frequency Coefficient Fusion}

The low-frequency subband reflects the approximate characteristics of the source image and it aggregates most information and energy of the image. Here, we improve the general average gradient $(A G)$ fusion rule to a new model to fuse the low frequency of the IR image (Image $\boldsymbol{A}$ ) and visible image (Image $\boldsymbol{B}$ ). We propose to use a cross-term average gradient (CG) to balance and adjust the contribution of the coefficients from a source image.

The general average gradient and cross-term average gradient of the image pixel can be described as:

$$
\begin{gathered}
A G=\frac{1}{(M-1)(M-1)} \sum_{i=1}^{M-1} \sum_{j=1}^{N-1} \sqrt{\frac{\left(\Delta I_{x}\right)^{2}+\left(\Delta I_{y}\right)^{2}}{2}} \\
C G=\frac{1}{(M-1)(M-1)} \sum_{i=1}^{M-1} \sum_{j=1}^{N-1} \sqrt{\left|\Delta I_{x}\right|\left|\Delta I_{y}\right|}
\end{gathered}
$$

The weight factors can be described as 
10.21611/qirt.2016.156

$$
\begin{aligned}
& W_{A}=\frac{1}{1+\eta_{l}}\left(\frac{A G_{A}}{A G_{A}+A G_{B}}+\eta_{l} \frac{C G_{A}}{C G_{A}+C G_{B}}\right) \\
& W_{B}=1-W_{A}
\end{aligned}
$$

The fused low-frequency coefficients can be expressed as $C_{\text {lowf }}=W_{A} C_{A}+W_{B} C_{B}$.

\subsection{High-frequency Coefficient Fusion}

The high-frequency subband reflects the salience and edge information from the source images. Here, we present to use the generalized Gaussian probability density function (GG-PDF) to approximate the marginal density of high-frequency coefficients. The GG-PDF can be defined as:

$$
\begin{gathered}
f(s ; \alpha, \beta)=\frac{\alpha}{2 \beta \Gamma(1 / \alpha)} e^{-(|s| \beta)^{\alpha}} \\
\Gamma(z)=\int_{0}^{+\infty} e^{-t} t^{z-1} d t,(z>0)
\end{gathered}
$$

where, $S$ is the coefficient value in an image location, $\beta$ is the scale parameter which models the width of the PDF peak, $\alpha$ is the shape parameter which is inversely proportional to the decreasing rate of the peak. After the formula derivation, it can be obtained that

$$
\begin{aligned}
& F(\alpha)=\frac{m_{1}^{2}}{m_{2}^{2}}=\frac{\Gamma^{2}(2 / \alpha)}{\Gamma(1 / \alpha) \Gamma(3 / \alpha)} \\
& \left\{\begin{array} { l } 
{ \hat { \alpha } = F ^ { - 1 } ( \frac { \hat { m } _ { 1 } ^ { 2 } } { \hat { m } _ { 2 } ^ { 2 } } ) } \\
{ \hat { \beta } = \hat { m } _ { 1 } \frac { \Gamma ( 1 / \hat { \alpha } ) } { \Gamma ( 2 / \hat { \alpha } ) } }
\end{array} \quad \left\{\begin{array}{l}
\hat{m}_{1}=\frac{1}{n} \sum_{i=1}^{n}\left|s_{i}\right| \\
\hat{m}_{2}=\frac{1}{n} \sum_{i=1}^{n}\left|s_{i}\right|^{2}
\end{array}\right.\right.
\end{aligned}
$$

From the estimation of $f(s)$, we can obtain the salience measures of the $\sigma_{A}$ and $\sigma_{B}$. Thus, the weight factors can be written as

$$
\begin{aligned}
& W_{A}=\frac{\sigma_{A}}{\sigma_{A}+\eta_{h} \sigma_{B}} \\
& W_{B}=1-W_{A}
\end{aligned}
$$

The fused high-frequency coefficients can be described as $C_{h i g h f}=W_{A} C_{A}+W_{B} C_{B}$.

\section{Preliminary Results}
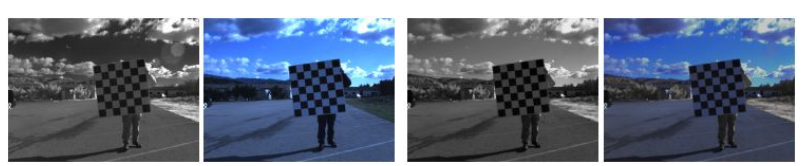

\section{REFERENCES}

[1] da Cunha, A. L., Jianping, Z., \& Do, M. N. (2006). The Nonsubsampled Contourlet Transform: Theory, Design, and Applications. Image Processing, IEEE Transactions on, 15(10), 3089-3101. doi: 10.1109/TIP.2006.877507.

[2] Xiuqiong Zhang, "Infrared and Color Visible Image Sequence Fusion Based on Statistical Model and Image Enhancement," in Advanced Computer Theory and Engineering, 2008. ICACTE '08. International Conference on , vol., no., pp.934-937, 20-22 Dec. 2008 